\title{
EXCESS CARRIER DYNAMICS IN SiGe ULTRA-THIN LAYERS*
}

\author{
E. Gaubas ${ }^{\text {a }}$ A. Uleckas ${ }^{\mathrm{a}}$, and E. Simoen ${ }^{\mathrm{b}}$ \\ ${ }^{a}$ Institute of Materials Science and Applied Research, Vilnius University, Sauletekio 10, LT-10223 Vilnius, Lithuania \\ E-mail: edmundas.gaubas@ff.vu.lt \\ ${ }^{\mathrm{b}}$ IMEC, Kapeldreef 75, B-3001 Leuven, Belgium
}

Received 22 September 2005

\begin{abstract}
The excess carrier decay kinetics, measured by microwave absorption and reflection techniques in dislocations-rich strainrelaxed SiGe layered structures, are analyzed. The simultaneous recombination and multi-trapping processes were revealed and their parameters were extracted. The recombination lifetime decreases with threading dislocations density, while multitrapping is characterized by a trapping coefficient $>10$.
\end{abstract}

Keywords: recombination and trapping, strain-relaxed buffer layers, microwave absorption transients

PACS: 61.72.Hh, 72.40.+w

\section{Introduction}

Formation of the strained silicon layers on a strainrelaxed SiGe buffer (SRB) is one of the modern techniques to enhance the carrier mobility for the strongly reduced channel lengths in advanced nano-electronics devices [1]. However, relaxation of the SRB also results in creation of the threading and misfit dislocations chaotic networks. Therefore, the issue of the significant impact of dislocations on the carrier recombination and transport properties remains of concern, when dislocations may cause a disordered structure with the specific carrier trapping and asymmetric diffusion phenomena. However, a role of dislocations is not understood well enough. Determination of the carrier recombination and transport characteristics dependent on the dislocations density is important when predicting the integral electrical parameters of devices fabricated in the SRB substrates. Moreover, specialized methods should be exploited in revealing the properties of ultra-thin layers residing on the top of a multi-layered structure and in resolving the parameters of these layers on the background of the thick silicon substrate.

The aim of this paper is to investigate dynamics of carrier recombination and transport processes dependent on dislocations density in strained $\mathrm{Si}(\mathrm{SSi})$ on SiGe SRBs. To separate the carrier recombination and transport parameters in the ultra-thin layers on the

\footnotetext{
* The report presented at the 36th Lithuanian National Physics Conference, 16-18 June 2005, Vilnius, Lithuania.
}

background of Si substrates, the specific techniques of the transient microwave absorption/reflection by free carriers (MWA/MWR) at small excitation depth as well as transient diffraction on reflective grating (TRG) were exploited. Peculiarities of $J-V$ characteristics caused by the disordered structure of SRBs were also examined.

\section{Samples and measurement techniques}

Two sets of samples were examined. The first one consisted of diode structures fabricated by utilizing the $8 \mathrm{~nm} \mathrm{SSi}$ grown on 250 or $350 \mathrm{~nm} \mathrm{SRB} \mathrm{lay-}$ ers with a Ge concentration of $22 \%$. Both $\mathrm{p}^{+} / \mathrm{n}$ and $\mathrm{n}^{+} / \mathrm{p}$ junctions were fabricated by standard implantations. A junction depth $\left(d_{\mathrm{j}}\right)$ of $50 \mathrm{~nm}$ was estimated from secondary ion mass spectrometry. The second set of samples consisted of as-grown wafers with a $d_{\mathrm{SRB}}=420 \mathrm{~nm}$ thick SRB layer containing different dislocation densities.

To record the recombination characteristics of the top layers in the ultra-thin multi-layered structures, the microwave reflection (MWR) and transmission (MWA) regimes [2] were combined. The excitation depth was controlled and varied by the excitation wavelength (532 and $350 \mathrm{~nm}$ ) through modulation of the absorption coefficient. The transients of the reflected or transmitted MW $\mathrm{cw}$ radiation and their absolute values depend on the light-induced absorption / conductivity modulation $(\Delta \sigma)$ from the excess carrier density $\Delta n(t), \quad$ varying 
with time $t$ after the 30 ps laser pulse. The signal voltage $U(t)=f(\Delta \sigma d) \propto \Delta n(t) d_{\mathrm{SRB}}$ was recorded by a digital oscilloscope. Short pulse excitation was utilized to prevent diffusive broadening of the excess carrier domain over the layered structure thickness $d_{\mathrm{SRB}}$. To probe the bulk recombination of ultra-thin layers, the injection regime was also controlled by excitation density. Then, the extent of the space charge regions estimated through the Debye length was kept significantly less than the initial excitation domain. Synchronously, the Debye radius of the dislocation surrounding space charge cylinder was modified. Therefore, the lifetimes attributed to the high injection level were really measured.

To separate the diffusion and recombination components, which can overlap in the MWA decays, a technique of transient diffraction on reflective grating (TRG) [3], induced by UV pulsed radiation, was employed. The lateral diffusion between grating "strips" in the light-induced gradient of excess carriers usually prevails over other grating erasure processes at the properly selected grating spacing. The grating erasure time $\tau_{\text {ge }}$ as a function of grating period $\Lambda$ was examined. To avoid the self-modulation effects and to resolve spatially the diffracted beams, the UV light induced grating was probed by a reference beam of the second harmonic $\left(\lambda_{\mathrm{pr}}=532 \mathrm{~nm}\right)$ radiation of the same laser. Peculiarities of the diffraction on TRG arise due to mixed (amplitude-phase, non-sinusoidal) type of grating, which is formed by the periodical reflection coefficient $(\Delta R$, an amplitude component) and local deformation relief modulation ( $B$, a phase component) [3]. The exposure and grating erasure characteristics were investigated to clarify the dominant mechanisms and to determine the recombinative and diffusive erasure times, $\tau_{\mathrm{ge} D}=f\left(\Lambda^{2} /\left(4 \pi^{2} D\right)\right)$, due to carrier diffusion with coefficient $D$.

The MWA/R and TRG characteristics were examined on the second set of the SRB wafers, which had a mirror-like top layer, while the back-side was used as the silicon reference. The MWA measurements at the oblique incidence of the excitation and probe beams were performed in the metallized device structures nearby the boundary of the diode area in the samples of the first set. The $J-V$ characteristics were also examined in this set of samples, comparing diodes fabricated in the SRB structure and $\mathrm{Si}$ reference diodes made by the same technology, to control the electrical characteristics in the SRB structures.

\section{Experimental results and discussion}

The MWA transients in the $250 / 350 \mathrm{~nm}$ SRB samples containing $\mathrm{p}^{+} / \mathrm{n}$ junctions are illustrated in Fig. 1(a), and qualitatively the same character of carrier decay variations has been determined in both $\mathrm{p}^{+} / \mathrm{n}$ and $\mathrm{n}^{+} / \mathrm{p}$ junctions. The decays are clearly twocomponential for short wavelength excitation, while the bulk excitation transient is nearly one-componential. The decay shape transforms into the hyperbolic-like one at UV excitation, when the photoresponse comes from the top SRB layers. The asymptotic constituent in the latter case is clearly longer than those for bulk and near-surface excitation regimes. At the short wavelength excitation a longer transient relaxation is observed in the p-well structure as compared with the n-well structure. The initial component of the excess carrier decay at UV excitation manifests the rate of recombination processes of the top layers of the investigated structures. The effective carrier lifetimes evaluated within the initial decay stage are in the time scale of 30-270 ns for n-well diodes and 300-900 ns for ptype SRB material, respectively.

Investigations of the non-metallized wafers (second set) were performed by exploiting the regime of the UV excitation. The MWR transients recorded at the same 30 ps pulsed excitation intensity are illustrated in Fig. 1(b) for the sample with dislocations density of $2 \cdot 10^{6} \mathrm{~cm}^{-2}$, comparing the decays obtained for the rough substrate surface (transient 1 ) and mirror-like top SRB layer (curve 2). The decay shape and rate clearly differs for SRB and Si surfaces. The small initial component can be explained by considering that the excess carrier density causes a modulation of the Debye length of the surface states on bare Si. However, the MWR transient integrated over the SRB layers acquires the hyperbolic-like shape. Qualitatively the same variations were obtained in SRB layers of the other samples containing higher densities of dislocations of the second set, while the values of effective recombination lifetimes varied with threading dislocations density. To verify that the MW probe depth is concentrated within the top SRB layer of the investigated structure at UV excitation and to examine peculiarities appearing due to the extent of the initial domain of excess carriers and its broadening, the MWA and MWR measurements have been complementary performed. The initial stages of the MWA and MWR transients measured in SRB layers nearly coincide, and the effective lifetime $\tau_{\text {in }}$ of the initial decay stage, measured by the MWR regime, is not shorter than that of the MWA. This proves that the fast 

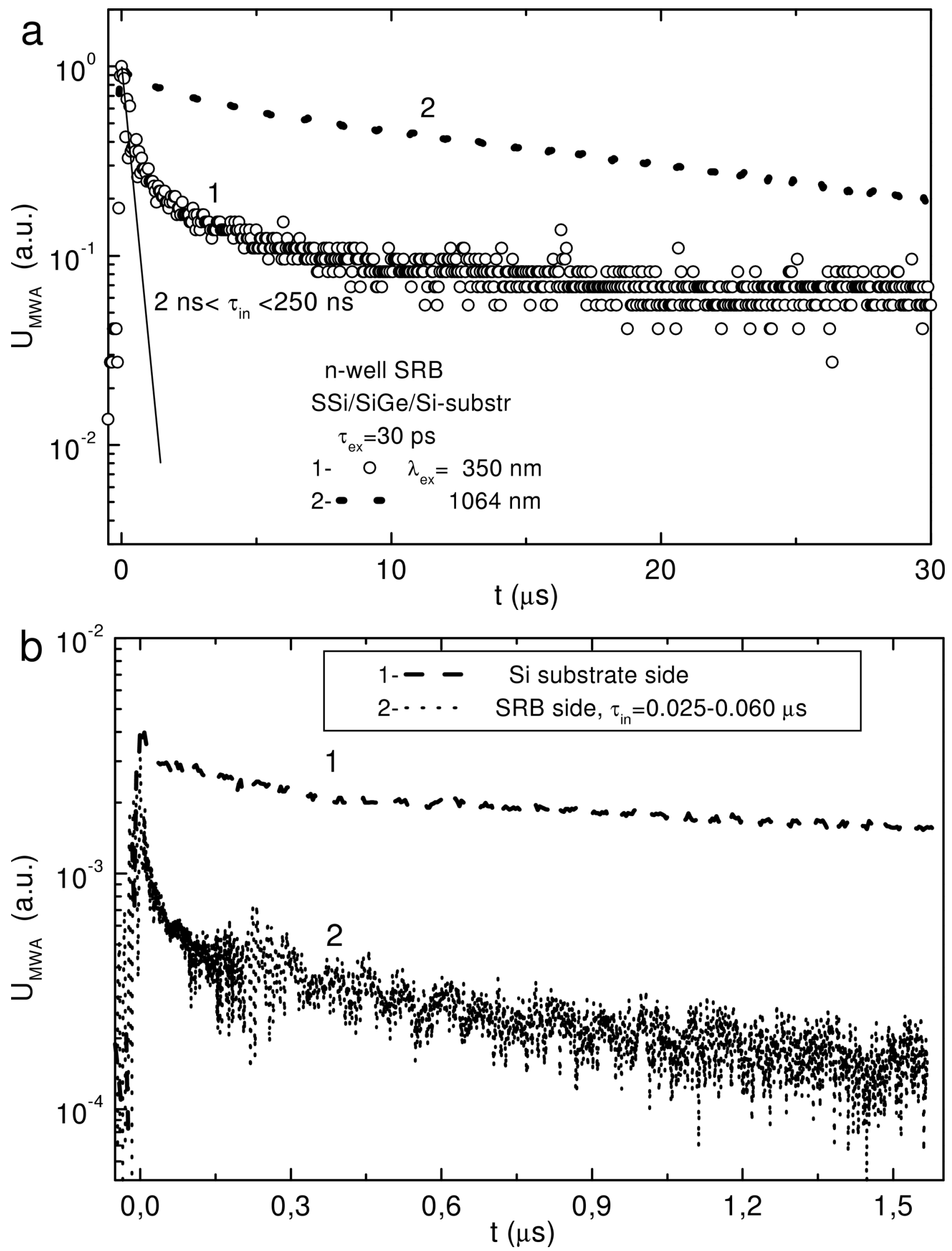

Fig. 1. (a) MWA transients in layered structure fabricated on a $350 \mathrm{~nm}$ SRB wafer, corresponding to $\mathrm{p}^{+} / \mathrm{n}$ junctions, for excitation wavelengths of 350 (1) and 1064 (2) nm. (b) The MWR relaxation in the Si substrate (1) is compared with that in the SRB layer (2), when measured by utilizing UV excitation of the same intensity. 


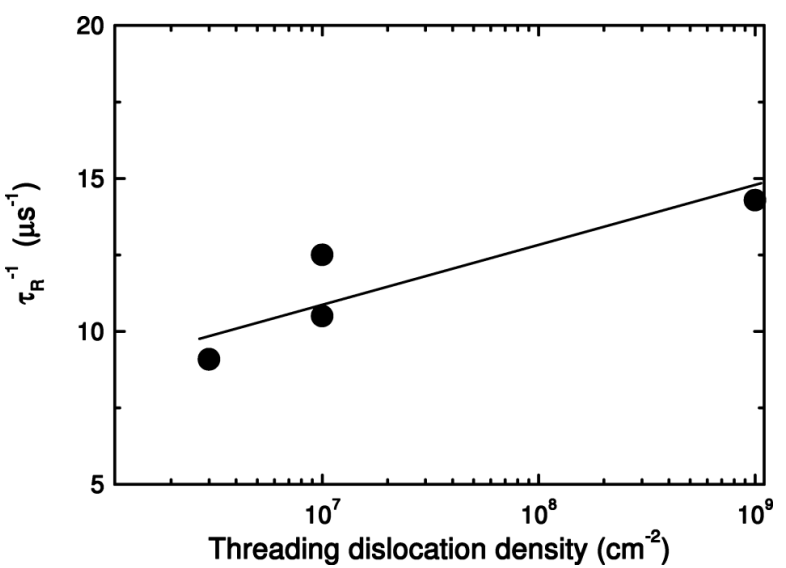

Fig. 2. Variations of the inverse recombination lifetime as a function of density of the threading dislocations in SRB layers measured by the MWA transient technique.

initial component of the MWA and MWR transients at UV excitation really integrates the carrier decay processes in the top or SRB layers, as in the case of MWR only the top density of carriers modifies the photoresponse.

The inverse effective lifetimes of the recombinative carrier decay as a function of dislocation density $N D D$ are presented in Fig. 2 for the as-grown SRBs. It can be deduced from Fig. 2 that threading dislocations serve as recombination centres, and the inverse recombination lifetime increases with their density. The density of dislocation-attributed recombination centres can be estimated as $N_{D}=N D D / C$ [4], where $C$ is the spacing between the broken bonds in a dislocation. This density $N_{D}$ is hereby evaluated to be equivalent to $4 \cdot 10^{13}-2 \cdot 10^{16} \mathrm{~cm}^{-3}$ for dislocation densities $N D D=$ $2 \cdot 10^{6}-10^{9} \mathrm{~cm}^{-2}$. Assuming an effective cross section of the order of $10^{-15} \mathrm{~cm}^{2}$ [4], the value of microscopic recombination lifetime $\tau_{\mathrm{R} \infty}$, attributed to high injection level, is estimated to be in the range of 0.005$2.5 \mu \mathrm{s}$ at room temperature, what is in good agreement with the effective lifetimes measured by MWA in the initial decay stages. However, the hyperboliclike asymptotic decay implies multi-trapping effects in the disordered structure. The influence of the multitrapping is most inherent for the asymptotic decay, after significant concentrations of excess carriers are captured into trapping levels attributed to threading dislocations (TD). Since the capture cross section and activation energy of these traps depend on their occupation and varies during relaxation of the excess carriers, the distribution of the trap depth in a dislocation cluster is random. The multi-trapping process seems to be more ductile to slower carrier diffusive transport in the disordered structure of dislocations. In the case of

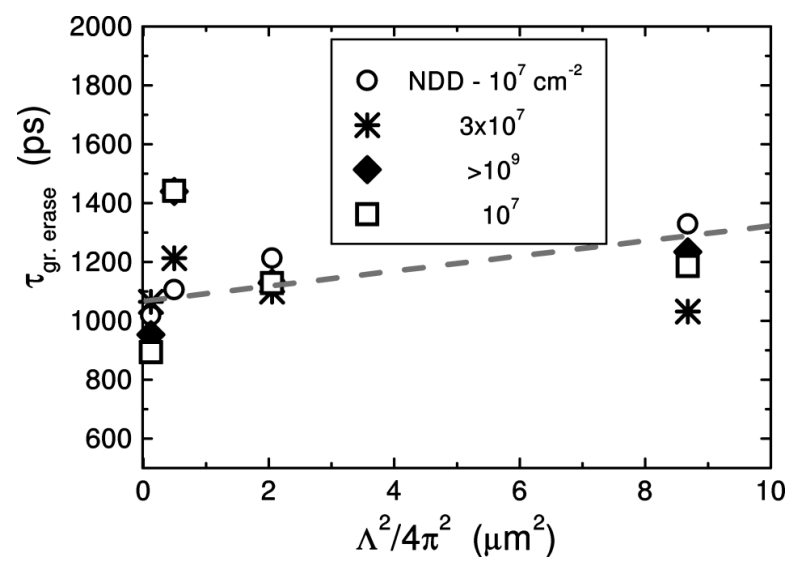

Fig. 3. Variation of the grating erasure times determined in SRBs, containing different dislocations densities, as a function of $\Lambda^{2} /\left(4 \pi^{2}\right)$.

competing recombination and trapping processes, the excess carrier decay is characterized by instantaneous lifetimes $\tau_{\text {inst }}$, expressed through a trapping coefficient $K_{\text {tr }}$ as $\tau_{\text {inst }}=\tau_{\mathrm{R} \infty} K_{\mathrm{tr}}\left(\tau_{\mathrm{tr}}\right)$. A simplified estimation of $K_{\text {tr }}$ can be made by extrapolating $\tau_{\mathrm{R} \infty}$ and taking the ratio $\tau_{\mathrm{R} \infty} / \tau_{\text {inst }}$. The extracted $K_{\mathrm{tr}}$ values were found to be rather large, $K_{\mathrm{tr}}>10$.

In the TRG experiments, the diffraction field of UVbeam self-diffraction as well as green light $532 \mathrm{~nm}$ probe beam diffraction contains few symmetrical (detectable and visible) diffraction beams only of the first order. Usage of the ps time scale pulses seems to limit more efficiently the evolution of the heating processes that cause the formation of the surface relief responsible for a phase grating component. The amplitude grating component is governed by modulation of the reflection coefficient. The recombination and transport rates were examined by employing the TRG erasure effective times measured by varying discretely the grating spacing in the range of $\Lambda=2-19 \mu \mathrm{m}$. Values of $\tau_{\text {ge }}$ were found to be in the time scale of 700-1500 ps for grating periods in the range of $\Lambda=2-19 \mu \mathrm{m}$. Then, the effective recombination lifetime is $\tau_{\text {eff }}=2 \tau_{\text {ge }}$, and its values $\geq 2-3$ ns correlate well with the time scale of the initial stages in the MWA and MWR transients. The grating erasure times in different samples as a function of $\Lambda^{2} /\left(4 \pi^{2}\right)$ are plotted in Fig. 3. Variation of the estimated grating erasure times with grating period is relatively slow. Thus, the recombination processes dominate in the grating erasure rate. In addition, the diffusion constituent in the grating erasure does not exceed the range of the experimental dispersion of the $\tau_{\text {ge }}$ values. The carrier ambipolar diffusion coefficient $D_{\text {a }} \leq 0.13 \mathrm{~cm}^{2} / \mathrm{s}$ can be only estimated from these $\tau_{\text {ge }}=f\left(\Lambda^{2}\right)$ plots in Fig. 3. The estimated $D_{\text {a }}$ value is 


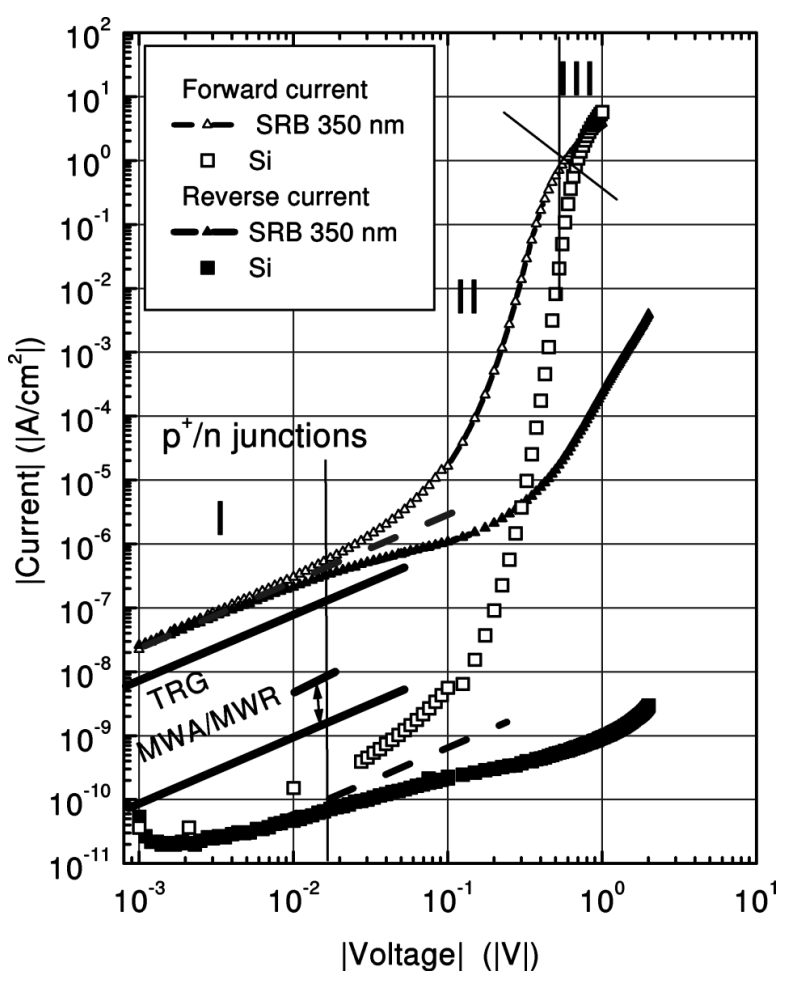

Fig. 4. Current density vs voltage characteristics of $\mathrm{p}^{+} / \mathrm{n}$ diodes. The solid lines indicate the possible relative shift of current values in SRBs respectively to the reference Si diode, evaluated using recombination lifetime values measured in the Si substrate and SRBs by using MWA/TRG methods. Ciphers indicate the ranges of quasi-linear $(I, I I I)$ and a super-linear $(I I)$ increase of current with voltage.

less than that for crystalline $\mathrm{Si}$ and $\mathrm{Ge}$, at least by an order of magnitude (e.g. $D_{\mathrm{a}} \approx 16 \mathrm{~cm}^{2} / \mathrm{s}$ in Si). Dislocations can be supposed to be screened at high excitation level, while small $D_{\text {a }}$ values $\ll 1 \mathrm{~cm}^{2} / \mathrm{s}$, inherent for amorphous materials [5], can be explained by the disorder of dislocations network. Thus, even in the case of medium dislocations density, when distances between dislocations are $\leq 6 \mu \mathrm{m}$, the crystalline carrier motion concept is hardly acceptable, since the distribution of dislocations is inhomogeneous. Thus, the TRG characteristics imply that the role of the diffusion in excess carriers decay is relatively small in the dislocation-rich SRB layers.

The recombination and trapping effects appear specifically in device structures, when the injected excess carriers are governed by the bias field. The dc $J-V$ characteristics measured in the $\mathrm{p}^{+} / \mathrm{n}$ metallized samples are illustrated in Fig. 4. Here, the $J-V$ characteristics obtained in the SSi-SRB junctions are compared with those determined in reference diodes fabricated by the same implantation conditions in crystalline $\mathrm{Si}$. The disordered, due to dislocations networks, SRB layered structure plays the most important role in the $J-V$ characteristics of the shallow junction diode. The geometry and type of contacts, diffusion current component in the vicinity of electrodes, and asymmetry of the contacts can be important factors in the range of small current densities. The double injection effects $[6,7]$ can be significant in the range of elevated voltages. Then, the structure can be modelled by regional approximations [6], when different regimes are inherent in separated layers (up to four characteristic regions), and role of these layers varies with applied voltage, it depends on the state of recombination and trapping centres. Thus, in such an intricate structure, the quantitative $J-V$ characteristics can be only simulated numerically. However, a qualitative resemblance of the experimental $J-V$ s measured in SRB diodes to those (Fig. 4 of Ref. [6]) modelled for space charge limited currents (SCLC) implies the importance of the carrier trapping, revealed in the MWA / MWR transients. Various ranges of $J-V$ can be separated in segments denoted in Fig. 4, and characterized by different slopes $J-V^{\alpha}$. Existence of the $J-V$ segment $J_{I I} \sim V^{m+1}$ can be an indication of appearance of double injection effects and of a role of the disorder of the structure $[6,8]$ with random spectrum of traps. Here, $\alpha=m+1$, and $m$ is an index of trapping dependence on applied voltage $V^{m}$. This also implies that concentration of traps is of $<10^{18} \mathrm{~cm}^{-3}$ at $d_{j} \leq 0.1 \mu \mathrm{m}$ [6]. The nominal trapping coefficient $K_{\mathrm{tr}, J-V}=N_{C M} / M_{\mathrm{tr}} \approx 6-10^{5}$ can be estimated assuming the parameters evaluated for SRBs: an activation energy $\Delta E_{M}=0.06-0.3 \mathrm{eV}$ for the dislocation levels, a bulk density $M_{\text {tr }}$ of dislocation-attributed centres $M_{\mathrm{tr}}=4 \cdot 10^{13}-2 \cdot 10^{16} \mathrm{~cm}^{-3}$, and an effective band density of states $N_{C M}$, ascribed to $M_{\mathrm{tr}}$, as $N_{C M}=2.5 \cdot 10^{16}-2.4 \cdot 10^{14} \mathrm{~cm}^{-3}$. This estimation is in qualitative agreement with parameters determined from MWA/MWR measurements. The relative shift of the recombination current in the linear $J-V$ range along the ordinate axis is estimated by exploiting the effective lifetime values measured directly in the initial segment of MWA and TRG transients. It is sketched by the solid lines in Fig. 4, relatively to that of the reference Si diode. This indicates that the measured shift of $J$ requires taking into account the trapping coefficients to superpose the increased current values. Thus, the features of carrier recombination-trapping in the SRB layers unveiled by transient techniques qualitatively correlate with the peculiarities of the $J-V$ dependences of diodes fabricated in the SRBs. 


\section{Conclusions}

The dynamic techniques based on the microwave probe (MWA and MWR) and light-induced reflection (TRG) have been demonstrated to be sensitive enough for the direct measurement of the carrier recombination and transport characteristics in ultra-thin layered structures in a non-invasive way. The recombination and trapping components were revealed in excess carrier decay transients. The high injection level recombination lifetimes from tens to hundreds of nanoseconds were measured in the SRB layers containing different densities of dislocations. A decrease of this lifetime as a function of the dislocation density was determined. However, the disorder caused by dislocations network and the stochastic carrier transport play the most important role. A hyperbolic-like relaxation of the asymptotic carrier decay part was determined in the SRBs and ascribed to multi-trapping processes characterized by trapping coefficients $K_{\mathrm{tr}}>10$. Value of the lateral carrier diffusion coefficient $D_{\mathrm{a}} \leq 0.13 \mathrm{~cm}^{2} / \mathrm{s}$, estimated from the TRG erasure time $\tau_{\text {ge }}$ as a function of the grating period $\Lambda^{2}$, is inherent for disordered structures. The character of the excess carrier recombination in the SRB layers unveiled by transient techniques qualitatively correlates with the peculiarities of the $J-$ $V$ dependences of diodes fabricated in the SRBs.

\section{Acknowledgements}

G. Eneman and R. Delhougne are acknowledged for the provided $J-V$ characteristics. R. Tomašiūnas is acknowledged for assistance in the TRG experiments.

\section{References}

[1] G. Eneman, E. Simoen, A. Lauwers, R. Lindsay, P. Verheyen, R. Delhougne, R. Loo, M. Caymax, P. MeunierBeillard, S. Demuynck, K. De Meyer, and W. Vandervorst, Analysis of junctions formed in strained $\mathrm{Si} / \mathrm{SiGe}$ substrates, MRS Proc. 809, 187 (2004).

[2] E. Gaubas, Transient absorption techniques for investigation of recombination properties in semiconductor materials, Lithuanian J. Phys. 43, 145 (2003).

[3] J. Vaitkus, E. Gaubas, and A. Rudaitis, Light induced reflective transient gratings on silicon surface, Litovskii Fizicheskii Sbornik 29, 578 (1989).

[4] H.F. Mataré, Defect Electronics in Semiconductors (Wiley-Interscience, New York, 1971).

[5] K. Jarasiunas, J. Vaitkus, E. Gaubas, L. Jonikas, R. Pranaitis, and L. Subacius, The diffraction of light by transient gratings in crystalline, ion-implanted and amorphous silicon, IEEE J. Quant. Electron. 22, 1298 (1986).

[6] M.A. Lampert and P. Mark, Current Injection in Solids (Academic Press, New York \& London, 1970).

[7] A.G. Milnes, Deep Impurities in Semiconductors (Wiley-Interscience, New York, 1973).

[8] S.V. Slobodchikov, K.M. Salikhov, and E.V. Rusu, Current transport and photoelectrical properties of $\mathrm{Pd}-\mathrm{SiO}_{2}-$ $\mathrm{n}(\mathrm{p})$-Si diode structures and their possible applications, Moldavian J. Phys. Sci. 2, 370 (2003).

\title{
PERTEKLINIŲ KRŪVININKŲ DINAMIKA YPAČ PLONUOSE SiGe DARINIUOSE
}

\author{
E. Gaubas ${ }^{\text {a }}$, A. Uleckas ${ }^{\text {a }}$, E. Simoen ${ }^{\text {b }}$ \\ ${ }^{\text {a } V i l n i a u s ~ u n i v e r s i t e t a s, ~ V i l n i u s, ~ L i e t u v a ~}$ \\ ${ }^{\mathrm{b}}$ IMEC, Leuven, Belgija
}

\begin{abstract}
Santrauka
Ištirti daugiasluoksniai itempto $\mathrm{Si}(\mathrm{SSi})$-SiGe-C-SiGe buferinių sluoksnių 8-400 nm storio dariniai, sudaryti ant kristalinio Si padèklų, kaitaliojant buferinio sluoksnio storį 250-420 nm tarpe ir dislokacijų tanki juose. Tirta nesalytiniais nenuostoviosios mikrobangu sugerties ir atspindžio metodais, kaitaliojant sužadinimo
\end{abstract}

gylį ir palyginant padèklo bei aktyvaus sluoksnio paviršius. Pasiūlyti tinkamiausi parametrų išskyrimo režimai, derinant sužadinimo spektrinę sriti, intensyvumą ir impulsų trukmę. Atskleisti nepusiausviruju krūvininkų tankio relaksacijos ypatumai, kuriuos lemia sparčioji rekombinacija ir laike ištęsti prilipimo vyksmai. 